\title{
Techniques
}

\section{On-Spot Brilliant Cresyl Blue Staining in Fine Needle Aspiration, a Novel Technique for Cytological Diagnosis}

\author{
Lateef Ahmad Sofi Rana K. Sherwani Mehboob Hasan Hage Nobin \\ Department of Pathology, Jawaharlal Nehru Medical College, Aligarh, India
}

\section{Key Words}

Fine needle aspiration cytology · Brilliant cresyl blue .

Rapid stain · Frozen section

\begin{abstract}
Objective: Application of on-spot staining with brilliant cresyl blue, a supravital stain, was investigated in fine needle aspirates from breast, lymph node and thyroid. Study Design: Patients with palpable breast lumps, lymph nodes and thyroid nodules were included in the study. Brilliant cresyl blue was used to stain the smears, which were evaluated for smear adequacy as well as cytological diagnosis. Smears were decolorized and subjected to Pap and/or HE staining for comparison. Results: Smears which were deemed hemorrhagic on the first attempt with brilliant cresyl blue correlated with Pap staining; then sites were reaspirated within minutes to obtain satisfactory cellular smears. Morphologically, cells which were designated malignant on supravital staining were mostly malignant on Pap/HE staining. Conclusion: On-spot brilliant cresyl blue staining increases the diagnostic potential on preliminary examination by decreasing the staining time and is as good as conventional stains in exhibiting cellular morphology.

(c) 2013 S. Karger AG, Basel
\end{abstract}

\section{Introduction}

Fine needle aspiration (FNA) is a simple, cost-effective, relatively less invasive and easy to perform technique gaining recognition and importance in the examination of lumps in various anatomical locations. Recently, ultrasound-guided FNA cytology (FNAC) has also been used for inaccessible locations, e.g. intra-abdominal and thoracic regions. FNA is fairly sensitive and specific in terms of distinguishing malignant cases from benign or reactive lesions and thus plays a major role in appropriate treatment strategies.

Hemorrhagic/inconclusive smears are one of the major shortcomings of FNA procedures. Quite often, reaspiration takes time and leads to a delay in diagnosis and treatment and also affects lab turnaround time. In India, this cytological technique is used extensively as it is a cheaper, less invasive and relatively easy technique for diagnosis of the vast majority of lesions of the breast, thyroid and lymph nodes.

Rapid staining and urgent reporting are cornerstones of clinical setups, particularly in the surgical setting, which can be equivalent to frozen section examination [1]. On spot (on-site) interpretation for sample adequacy

\section{KARGER}

E-Mail karger@karger.com

www.karger.com/acy
(C) 2013 S. Karger AG, Basel

0001-5547/13/0576-0641\$38.00/0
Correspondence to: Dr. Lateef Ahmad Sofi

Department of Pathology

Jawaharlal Nehru Medical College

Aligarh, UP 202002 (India)

E-Mail drslateef.2k7@gmail.com 


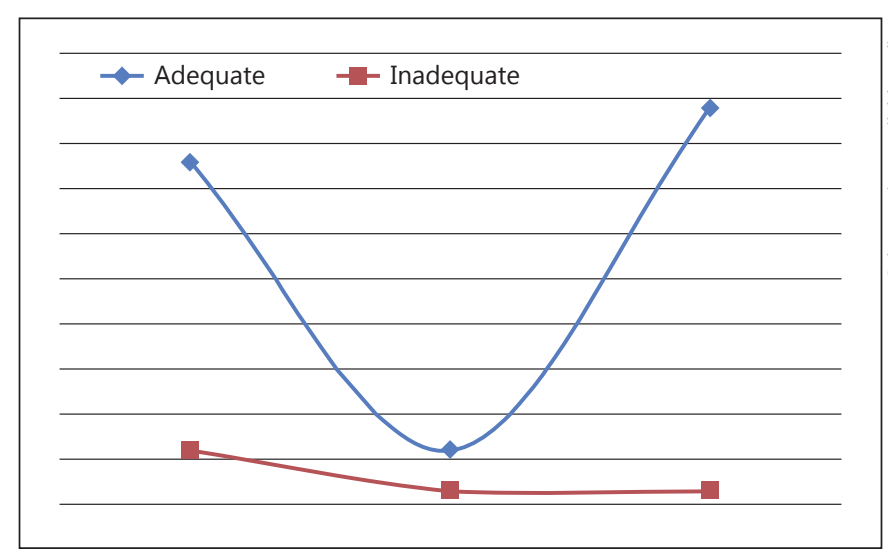

Fig. 1. Adequacy assessment before and after reaspiration with LIST.

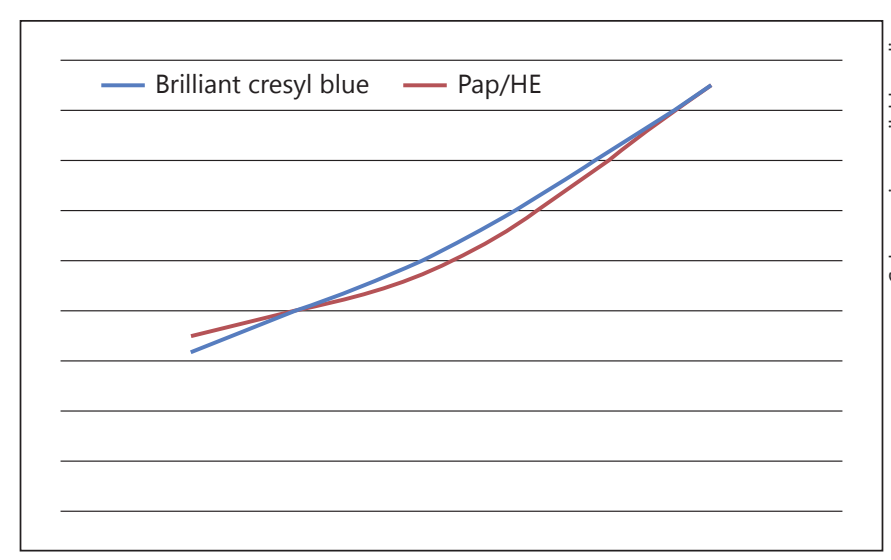

Fig. 2. Comparative diagnostic efficacy of brilliant cresyl blue and $\mathrm{Pap} / \mathrm{HE}$ staining.

Table 1. Adequacy assessment before and after reaspiration with LIST

\begin{tabular}{llc}
\hline & Adequate & Inadequate \\
\hline First aspiration & 76 & 12 \\
Reaspiration & 12 & 3 \\
After reaspiration & 88 & 3 \\
\hline
\end{tabular}

Table 2. Comparison between brilliant cresyl blue and Pap/HE staining

\begin{tabular}{lcc}
\hline Cytological diagnosis & Brilliant cresyl blue & Pap/HE \\
\hline Malignant & 32 & 35 \\
Benign/reactive & 53 & 50 \\
Inadequate & 3 & 3 \\
Total & 88 & 88 \\
\hline
\end{tabular}

in FNA smears improves diagnostic yield even in ultrasound-guided FNA $[2,3]$.

Aspiration attempts may not yield satisfactory cellularity (hemorrhagic/scant cellularity) depending upon the experience of the technician and the size, location and vascularity of the lump. Rapid staining techniques like toluidine blue and Diff-Quik are used to decide whether to reaspirate the site for satisfactory cellularity or for additional material for any special studies. Brilliant cresyl blue (1\%) is a supravital stain and has not been used previously as a rapid stain for evaluating specimen adequacy, unlike toluidine blue.

Reaspiration at the first visit, following an adequacy assessment by rapid staining, limits visits and shortens the time to definitive treatment, thus critically affecting morbidity. In the long run it saves time, money and manpower.

Smears stained with brilliant cresyl blue, without prior fixation (immediately after air drying), gave excellent details of adequacy as well as cell morphology in less cellular regions (groups and clusters). Since it is a supravital stain, nuclear and nucleolar details were as good as on Pap-stained smears.

\section{Material and Methods}

This study included a total of 88 cases, referred from OPD for FNAC. FNA was performed with a 23 -gauge needle. Slides were unfixed, stained with cresyl blue and examined and reviewed.

The composition of brilliant cresyl blue (1\%) was as follows: $1 \mathrm{~g}$ of brilliant cresyl blue, $0.7 \mathrm{~g}$ of sodium chloride, $0.6 \mathrm{~g}$ of sodium citrate and $100 \mathrm{ml}$ of distilled water.

Lateef s instant staining technique (LIST) is performed as follows:

- take the unfixed smear (air dried) immediately after aspiration;

- pour $100 \mu \mathrm{l}$ of brilliant cresyl blue (1\%) onto the slide;

- cover with cover slip;

- drain excess stain using filter paper;

- gently wipe under the surface of the slide with tissue paper;

- evaluate under a microscope for adequacy and preliminary diagnostic impression;

- repeat aspiration if cellularity is inadequate or hemorragic;

- if adequate, gently reimmerse the slide in absolute alcohol (to prevent loss of material due to fluid currents), and the cover slip will fall off by itself;

- for more rapid decolorisation, acid alcohol may be employed;

- stain using Pap or HE;

- compare the findings.

\section{Results}

On the first aspiration attempt, $13.6 \%$ of cases (12/88) were deemed unsatisfactory, as assessed by LIST. A repeat FNAC (reaspiration) at the same visit showed satisfactory 
3

Fig. 3. Adequate cellularity with brilliant cresyl blue.

Fig. 4. Adequate cellularity in a breast lump.

Fig. 5. Cellular morphology in a lymph node aspirate.

Fig. 6. Cellular morphology in a breast lump.
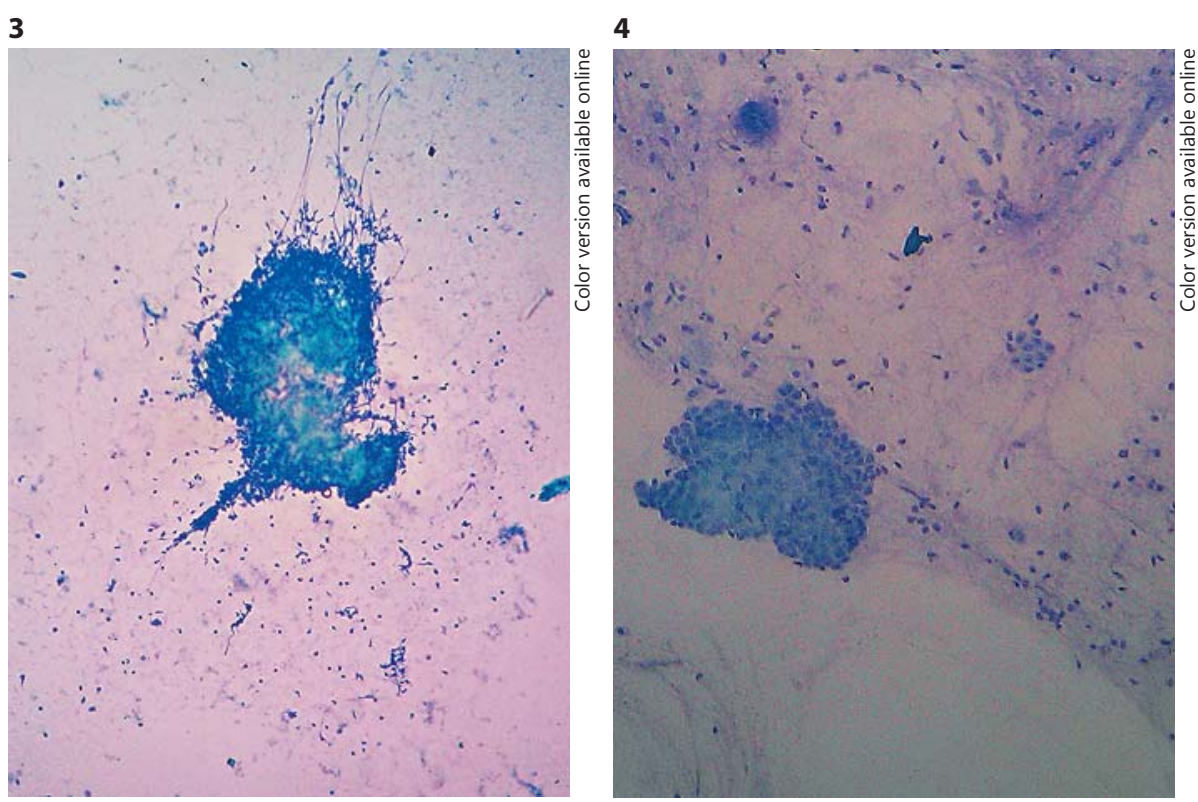

\section{5}
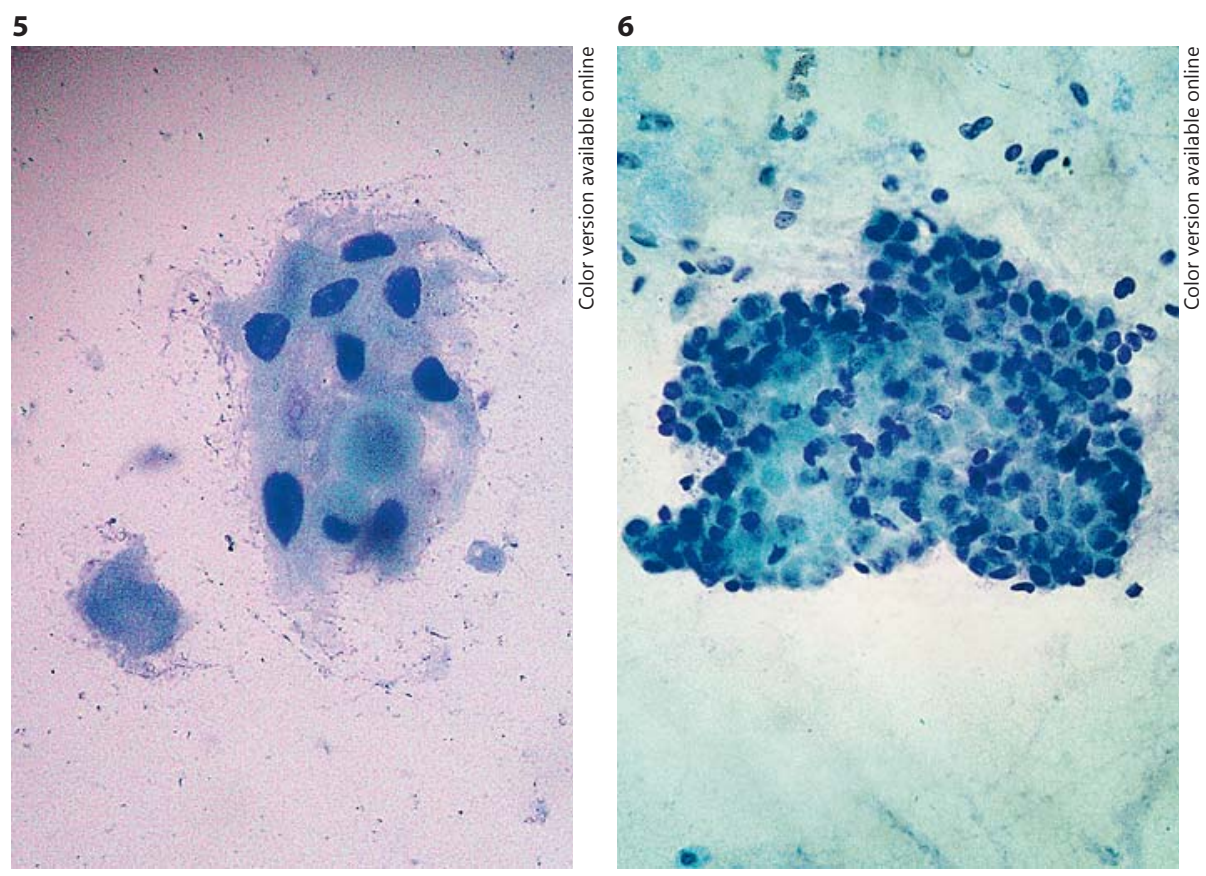

cellularity in $96 \%$ of cases $(85 / 88)$; however, a few cases $(3.4 \%, 3 / 88)$ remained hemorrhagic/unsatisfactory (table 1 ; fig. 1).

A majority of the cases (approx. 95\%) appearing malignant with brilliant cresyl blue staining correlated with Pap and/or HE staining (table 2; fig. 2). Three cases were missed owing to an intense inflammatory infiltrate obscuring cell morphology.

On-Spot Brilliant Cresyl Blue Staining in FNA

\section{Discussion}

FNA is currently employed for the diagnosis of lymphadenopathy, breast lumps and thyroid nodules. It is the initial investigation of choice for the above-mentioned swellings. Being cost-effective with good sensitivity and specificity, it is a preferred method for preoperative diagnosis.

Quick staining techniques are used in many centers for cytological assessment, e.g. Diff-Quik for air-dried smears 


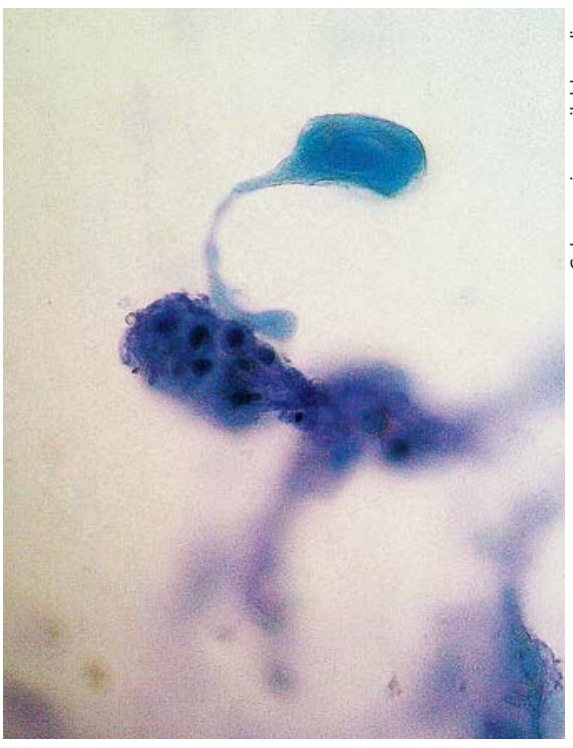

Fig. 7. Squamous cell carcinoma in a lymph node.

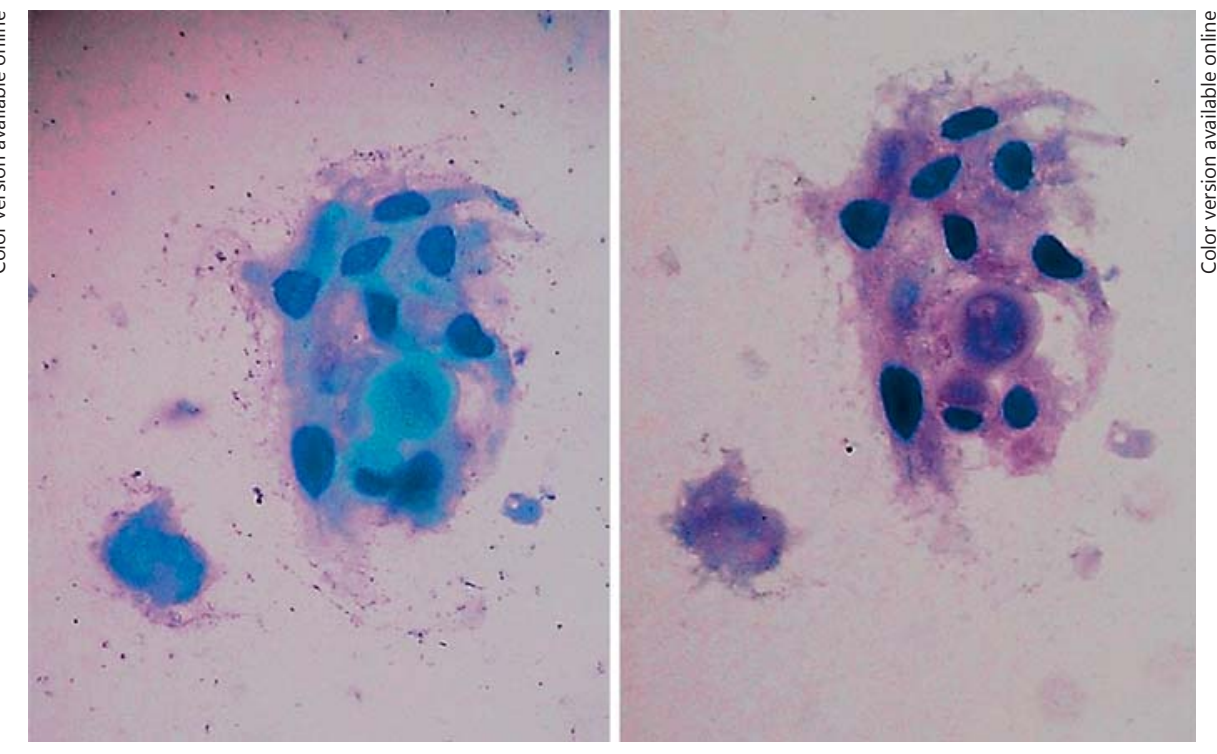

Fig. 8. Cytological comparison between LIST and HE. and toluidine blue for alcohol-fixed smears. Our study employed unfixed fresh FNA smears, and staining was almost instant, in a procedure we called Lateef's instantaneous staining technique (LIST), with which smears can be seen within $30 \mathrm{~s}$ of adding the stain (20-45 s).

Ammanagi et al. [1] recently investigated the role of toluidine blue for rapid staining in assessment of aspiration adequacy and reported an improvement in the efficiency of FNA. Borget et al. [4] concluded that on-site staining should dramatically reduce the rate of failures and thus enable same-day reporting and repeat sampling. Wong and $\mathrm{Di}$ [5] studied the role of toluidine blue-ethanol to determine aspiration adequacy and make a preliminary evaluation followed by correlation with HE. Howell et al. [6] used toluidine blue on alcohol-fixed slides and examined them immediately for adequacy. Similarly, Kamal et al. [7] studied breast lumps using ultrafast Pap for rapid assessment for adequacy and intraoperative FNA. Canil et al. [8] studied the role of toluidine blue in frozen rectal biopsy sections and noted that toluidine blue is superior to $\mathrm{HE}$ in detecting ganglion cells.

Our study employed brilliant cresyl blue, which is a cheap and easily available supravital stain, usually used in a hematological setting for reticulocyte count. Results from our study are promising in terms of adequacy assessment (fig. 3, 4). Cytological morphology in less crowded areas and cellular differentiation were excellent (fig. 5, 6). Nuclear and nucleolar details were as good as with Pap. A positive diagnosis of malignancy could be given on a preliminary basis, although intense inflammation obscuring cell morphology stains all areas gray. A large-scale study may further increase accuracy. For correlation, slides were further subjected to Pap or HE staining (fig. 7, 8).

This technique decreases the need for revisiting for reaspiration, thus increasing lab turnover as well as the diagnostic yield.

\section{Conclusion}

The instant staining technique described herein is a cost-effective, reliable method for adequacy assessment of FNA smears. It is one of the fastest staining methods and can easily be used to rule out a malignant swelling, being as good as conventional stains in exhibiting cellular morphology. The staining protocol is not cumbersome and can be performed even in small clinical setups.

\section{Acknowledgements}

We are highly thankful to our department for funding and supporting this study. Special thanks to Mr. Chand Mian, senior scientific officer in the cytopathology lab, for his cooperation and assistance. 


\section{References}

1 Ammanagi AS, Dombale VD, Patil SS: Onsite toluidine blue staining and screening improves efficiency of fine-needle aspiration cytology reporting. Acta Cytol 2012;56:347351.

2 Alsohaibani F: Does onsite cytotechnology evaluation improve the accuracy of endoscopic ultrasound guided fine needle aspiration biopsy? Cancer J Gastroenterol 2009;23: 26-30.

$>3$ Liew P-L, Liu T-J, Hseih M-C, Lin H-P, Lu C-F, Yao M-S, Chen C-L: Rapid staining and immediate interpretation of fine needle aspi- ration cytology for palpable breast lesions: diagnostic accuracy, mammographic, ultrasonograhic and histopathological correlation. Acta Cytol 2011;55:30-37.

$>4$ Borget I, Vielh P, Leboullex S, et al: Assessment of the cost of fine needle aspiration cytology as a diagnostic tool in patients with thyroid nodules. Am J Clin Pathol 2008;129: $763-771$.

5 Wong NL, Di F: Psuedosarcomatous fasciitis and myositis. Diagnosis by fine needle aspiration cytology. Am J Clin Pathol 2009;132: 857-865.
6 Howell LP, Gandour-Edwards R, Folkins K, Davis R, Yasmeen S, Afify A: Adequacy evaluation of fine needle aspiration biopsy in breast clinic setting. Cancer 2004;102:295-301.

7 Kamal MM, Bodelle A, Munshi MM, Bobhate SK, Kher AV: Efficacy of a modified Ultra Fast Papanicolaou stain for breast aspirates. Indian J Pathol Microbiol 2000:43:417-421.

8 Canil M, Meir K, Jevon G, Sturby T, Moerike S, Gomez A: Toluidine blue staining is superior to $\mathrm{H}$ \& $\mathrm{E}$ staining for identification of ganglion cells in frozen rectal biopsies. Histologic 2007;40:1-3. 